\title{
Componentes de la variabilidad espacial en el manejo por sitio específico en banano
}

\author{
Darío Antonio Castañeda Sánchez ${ }^{(1)}$, Daniel Francisco Jaramillo Jaramillo(2) y José Miguel Cotes Torres ${ }^{(2)}$
}

(1)Politécnico Colombiano Jaime Isaza Cadavid, Carrera 48, № 7-151, Medellín, Colombia. E-mail: dacastaneda@elpoli.edu.co (2)Universidad Nacional de Colombia, Sede Medellín, Calle 59A, no 63-20, Medellín, Colombia. E-mail: djaramal@unal.edu.co, jmcotes@unal.edu.co

Resumen - El objetivo de este trabajo fue evaluar la variabilidad espacial de la producción de banano en función de variables físicas y químicas del suelo y de las características fisiográficas de la finca, con miras a seleccionar aquellas con mayor potencial de uso en programas de manejo por sitio específico. Se georreferenciaron 130 unidades productivas de banano clon Williams (Cavendish AAA) distribuidas en cuatro lotes de la finca y tres unidades de suelo. Se determinó el peso del racimo y la cantidad de raíz funcional, para cada planta, y 35 variables físicas y químicas del suelo. Se relacionó la variabilidad espacial de la producción en función de las variables del suelo, a partir de cuatro estrategias: ejes coordenados como covariables; variables físicas y químicas del suelo y raíz funcional como covariables; división del análisis por lotes; y división del análisis por unidades de suelo. La división por lotes resultó ser la mejor estrategia para modelar la variabilidad espacial de la producción de banano. El análisis por este modelo permitió establecer grupos de variables del suelo que se relacionaron significativamente y explicaron más del $69 \%$ del peso de los racimos de banano dentro de cada lote. Estos grupos de variables son los de mayor potencial para el establecimiento de un programa de manejo por sitio específico.

Términos para indexación: Musa acuminata, agricultura de precisión, geoestadística, tendencia espacial, variograma.

\section{Components of the spatial variability in site-specific management in banana}

\begin{abstract}
The objective of this work was to evaluate the spatial variability of banana production, in function of physical and chemical variables of soil and of farm physiographic characteristics, in order to select those with the greatest potential for use in site-specific management program. One hundred thirty productive units of banana clone Williams (Cavendish AAA) distributed in four lots and tree soils units of the farm were georeferenced. The bunch weight and root functionality were determined for each plant, as well as 35 physical and chemical soil variables. The spatial variability of the production was evaluated in function of these soil variables, using four different strategies: axes as covariates; both physic and chemical soil properties as covariates; analysis made by individual plot; and analysis made by soil unit. The individual plot study was the best strategy to model the spatial variability of banana production. This analysis model allowed to set soil variable groups which were significantly correlated and precisely explained more than $69 \%$ of the bunch weight inside the plots. These groups of variables are the ones with higher potential for the establishment of a site-specific management program.
\end{abstract}

Index terms: Musa acuminata, precision agriculture, geostatistics, spatial trends, variogram.

\section{Introducción}

La existencia de variabilidad espacial en los rendimientos se ha demostrado en banano (Stoorvogel \& Vargas, 1998) y otros cultivos de interés (Diker et al., 2004; Ping \& Dobermann, 2005; Miao et al., 2006; Amado et al., 2007). En el cultivo de banano, para una misma finca, se han encontrado áreas con producciones entre 41,4 y 63 toneladas ha-1 por año de banano tipo exportación, y se estima que las extracciones de nutrientes requeridas para esas producciones varían en 35\% (Stoorvogel \& Vargas, 1998).

En cultivos como algodón, soya, maíz y trigo, se ha encontrado autocorrelación en la magnitud de la producción de acuerdo a la distancia entre observaciones (Long, 1998; Kravchenko \& Bullock, 2000; Voortman \& Brouwer, 2003; Amado et al., 2007). La variabilidad de los rendimientos del sistema aumenta conforme la escala de trabajo, los factores de rango amplio como la topografía, la clase de suelo,

Pesq. agropec. bras., Brasília, v.45, n.8, p.836-845, ago. 2010 
la precipitación, la temperatura, la distribución de la profundidad de los niveles freáticos, los sistemas de manejo del suelo y del cultivo, y las unidades experimentales (Zhang et al., 2002; Amado et al., 2007). Así, Kravchenko \& Bullock (2000) encontraron que el efecto acumulado de las variables topográficas era responsable del $20 \%$ de la variabilidad de la producción de maíz y soja. Blackmore (1999) investigó el efecto de la tendencia espacial y temporal sobre los rendimientos, en sistemas de trigo y colza aceitera, manejados como monocultivo y en asocio durante seis años, y encontró que el área de estudio, de acuerdo con los rendimientos, podría clasificarse en tres categorías: áreas de monocultivo con alta o baja producción pero estable y las áreas con trigo y colza asociados con rendimientos inestables.

Estos factores de variación de mayor rango forman áreas en las cuales el promedio de la variable no es homogéneo o es no estacionario en todas sus unidades. Cuando una variable es no estacionaria, autores como Lambert et al. (2004), Jaramillo (2006), Diggle \& Ribeiro (2007) y Sadler et al. (2008) argumentan que la variable tiene tendencia espacial y sugieren que una forma de extraerla es a través de la descomposición de la variabilidad por fuentes desconocidas, tales como los ejes coordenados, o por covariables como características químicas y físicas del suelo asociadas a esta tendencia.

Para Gauggel et al. (2003), en el cultivo de banano, los factores que dañan el sistema radical de la planta son los mismos que determinan la producción y, en estos, las variables fisicoquímicas del suelo tienen un papel importante. El uso racional de insumos, en el cultivo de banano, requiere entonces una precisa determinación de la variación espacial de las variables edáficas asociadas con los rendimientos. Sin embargo, esta no es una estrategia común en las fincas bananeras, donde se realiza, en la mayoría de los casos, un manejo uniforme de toda la finca, lo que lleva a un uso de fertilizantes excesivo en unos casos y deficiente en otros.

El propósito de este trabajo fue el de modelar, descomponer y explicar la variabilidad espacial de la producción de banano, como peso del racimo, en términos de las variables físicas, químicas y fisiográficas - como unidades de suelos y lotes relevantes -, con miras a seleccionar las variables de mayor potencial de caracterización espacial para ingreso en un programa de manejo por sitio específico.

\section{Materiales y Métodos}

La investigación se llevó a cabo entre febrero de 2007 y abril 2008, en seis hectáreas del campo experimental, propiedad de la Asociación de Bananeros de Colombia, ubicada en el Municipio de Carepa, región del Urabá antioqueño, a $7^{\circ} 46^{\prime} 45^{\prime \prime} \mathrm{N}$ y $76^{\circ} 40^{\prime} 22^{\prime \prime} \mathrm{W}$. La región presenta clima cálido húmedo, correspondiente a la zona de vida de bosque húmedo tropical $(\mathrm{bh}-\mathrm{T})$. El área de la investigación se distribuyó en tres lotes: L3, L4 y L5, según división y nomenclatura del campo experimental, todos sembrados con banano (Musa acuminata) clon Williams (Cavendish AAA), en cuadro y a distancias entre plantas y surcos de $2,5 \mathrm{~m}$, desde el año 2005. A esta área se le hizo un levantamiento planimétrico, en el que se ubicaron los lotes y el sistema de drenajes, con un GPS marca Trimble GeoXT estandarizado, bajo el sistema de proyección UTM WGS 84 zona $18 \mathrm{~N}$. El campo experimental se ubica en la llanura aluvial del piedemonte de la Serranía de Abibe, con un relieve plano, con pendiente menor que $3 \%$.

Los lotes en el campo experimental están delimitados por los canales de drenaje del nivel secundario. Adicionalmente, el lote L4 es atravesado diagonalmente por un canal de drenaje con un tamaño mayor que los secundarios, razón por la cual ese lote se dividió en dos, y sus áreas se identificaron como L4A y L4B, en este estudio, para un total de cuatro lotes (Figura 1 A). El campo seleccionado se asienta en tres clases de suelos (United States Department of Agriculture, 2006) (Figura 1 B): la consociación Fluventic Eutrudepts fina (A), de 0,9 ha, con el 12\% de la población de plantas evaluadas; la consociación Fluvaquentic Eutrudepts franca fina sobre arcillosa (B), de 3,3 ha, con el 73\% de la población; y la consociación Vertic Endoaquepts franca fina (C), de 1,8 ha, con el $15 \%$ de la población de plantas.

Se georreferenciaron en cuadrícula con celdas de 20x20m, con el GPS descrito anteriormente, 130 unidades productivas de banano, cada una representada por tres plantas de generaciones diferentes. Las plantas de la tercera generación, en cada unidad, fueron estandarizadas por la selección de yemas de la primera corona del cormo, con un tamaño menor que $5 \mathrm{~cm}$ de altura, habiéndose eliminado las demás. Estas plantas se llevaron hasta producción, y se registró el peso total del racimo en kilogramos (PR) para cada una de ellas.

Cuando el $50 \%$ de las plantas seleccionadas emitieron su inflorescencia, debido a que es una 
fase fenológicamente visible y de alta extracción de nutrientes, en cada sitio donde se marcaron las plantas se tomaron muestras disturbadas o no, para la evaluación de características químicas y físicas del suelo y de cantidad de raíces. En campo, entre 0 y $5 \mathrm{~cm}$ de profundidad, se determinó la resistencia a la penetración del suelo a 30, 60 y $100 \mathrm{~cm}$ de distancia de la planta en evaluación - CP30, CP60 y CP100 -, con un penetrómetro de bolsillo Soiltest (Soiltest, Nueva Delhi, India). Con un cilindro metálico biselado, con diámetro de 4,3 cm y altura de $4,83 \mathrm{~cm}$, se evaluó la densidad del suelo (Da), a la distancia de $30 \mathrm{~cm}$ de la planta y entre 5 y $10 \mathrm{~cm}$ de profundidad. Se tomó una muestra sin disturbar de $200 \mathrm{~g}$ aproximadamente, a $30 \mathrm{~cm}$ de la planta y entre 0 y $15 \mathrm{~cm}$ de profundidad. En laboratorio, se determinaron tanto en seco como en húmedo los siguientes índices de estabilidad estructural: el diámetro ponderado medio (DPMS y DPMH); el índice de estructuración (IES y IEH); la cantidad de agregados finos ( $<0,5 \mathrm{~mm}$ de diámetro) (AFS y AFH); y la cantidad de agregados extremos $(>2 \mathrm{~mm}$ y $<0,5 \mathrm{~mm}$ de diámetro) (AES y AEH). También se evaluó, con parte de esta misma muestra, la retención de humedad gravimétrica del suelo a capacidad de campo $(0,3 \mathrm{~atm})$ $\mathrm{y}$ en el punto de marchitez permanente (15 atm) (H0,3 y H15, respectivamente). Adicionalmente, se tomó una muestra disturbada con el barreno, de $1 \mathrm{~kg}$ aproximadamente, compuesta de cuatro submuestras, ubicadas a $30 \mathrm{~cm}$ de distancia de la planta marcada, en direcciones opuestas y ortogonales y a una profundidad entre 0 y $15 \mathrm{~cm}$, sobre la cual se determinaron, en laboratorio, la textura (\%) - arena (A), limo (L) y arcilla (Ar) -, la cantidad de arcilla dispersa (ArD, \%), y el coeficiente de dispersión (CD). Se determinaron también las variables químicas: $\mathrm{pH}$; contenido de materia orgánica (MO, \%); la capacidad de intercambio catiónico a pH del suelo (CICE, $\mathrm{cmol}_{\mathrm{c}} \mathrm{kg}^{-1}$ ) y a $\mathrm{pH} 7$ (CIC7); los contenidos intercambiables $\left(\mathrm{cmol}_{\mathrm{c}} \mathrm{kg}^{-1}\right)$ de $\mathrm{Ca}, \mathrm{Mg}, \mathrm{K}$ y Al; y los contenidos disponibles ( $\mathrm{mg} \mathrm{kg}^{-1}$ ) de $\mathrm{P}, \mathrm{S}, \mathrm{Fe}, \mathrm{Mn}, \mathrm{Cu}, \mathrm{Zn}$ y B, de acuerdo con los métodos descritos en Motta (1990).

Para la evaluación de raíces, en cada uno de los 130 puntos anteriormente descritos, ubicados sobre la malla establecida y a una distancia de $30 \mathrm{~cm}$ de la planta marcada, se hizo un hoyo de $18 \mathrm{~cm}$ de largo, ancho y profundidad, del cual se extrajo el suelo con todas las raíces de banano encontradas en él; se estableció la cantidad de raíces totales (RT) en g $100 \mathrm{~g}^{-1}$, y se discriminó el porcentaje de raíces vivas o funcionales (RF), según Calvo \& Araya-Vargas (2001) y Araya-Vargas (2002).

El análisis estadístico usó como variable respuesta el peso de los racimos, y como covariables las coordenadas, las propiedades del suelo y el contenido de raíces funcionales. La inferencia acerca de la homogeneidad de varianzas y de medias, para el peso de los racimos, se efectuó por pares de grupos formados de acuerdo a los lotes y a las unidades de suelo, con los estadísticos de prueba $\mathrm{F}_{0} \mathrm{y}$ t para las varianzas y las medias, respectivamente, con nivel de confianza del 95\%. Se verificó normalidad para peso de los racimos, a través de la prueba de Shapiro-Wilks.

Posteriormente, se seleccionaron las covariables de suelo que más se relacionan con el peso del racimo, por el algoritmo paso a paso "stepwise", basado en el criterio de comparación de Akaike (AIC, por sus siglas en inglés) e implementado en el paquete $\mathrm{R}$ (R Development Core Team, 2009). Esto se realizó para todas las muestras y en subgrupos por lote y unidades de suelo. Se utilizaron lotes para controlar las posibles variaciones por el manejo agronómico del cultivo, debido a que el banano es un cultivo intensivo, mientras que se utilizaron las clases de suelo con el fin de controlar las posibles diferencias debidas al tipo de suelo.

Los procedimientos geoestadísticos, en toda el área investigada $(\mathrm{n}=130)$, se efectuaron con la evaluación del comportamiento espacial del peso de racimo en función de las covariables significativas, obtenidas anteriormente, y de los ejes coordenados como estrategias de extracción de la tendencia. Para estos análisis se utilizó el paquete geoR (Ribeiro Junior \& Diggle, 2001).

La dependencia espacial del peso de racimos, para todo el campo $(\mathrm{n}=130)$, se evaluó con el variograma, en las siguientes direcciones: omnidireccional y para los ángulos de $0,30,60$ y $90^{\circ}$. Posteriormente, se corrigió la anisotropía con la transformación de los ejes de acuerdo con el ángulo y la relación de anisotropía encontrada, y con la función de transformación geométrica implementada en el paquete geoR (Ribeiro Junior \& Diggle, 2001). Finalmente, se modeló la variabilidad espacial del peso de los racimos, teniéndose en cuenta cada uno de sus componentes (Lark, 2002; Diggle \& Ribeiro Junior, 2007). 


\section{Resultados y Discusión}

Los gráficos de burbujas muestran la ubicación espacial, en el campo experimental, del peso de los racimos, delimitada por los lotes y por las unidades de suelo (Figuras 1 A y B). Nótese que sobre la diagonal del campo tienden a concentrarse las plantas que produjeron racimos con mayor magnitud en el peso $\mathrm{y}$, hacia los vértices laterales, los de menor peso, lo que evidencia una tendencia espacial. El valor p, para la relación de varianzas de los pesos de los racimos entre los lotes, indica que la varianza del lote L4B es diferente de la del lote L4A ( $p=0,023)$; en los demás casos, no se encontró diferencia significativa entre varianzas. Con respeto al tipo de suelo, la varianza muestral de
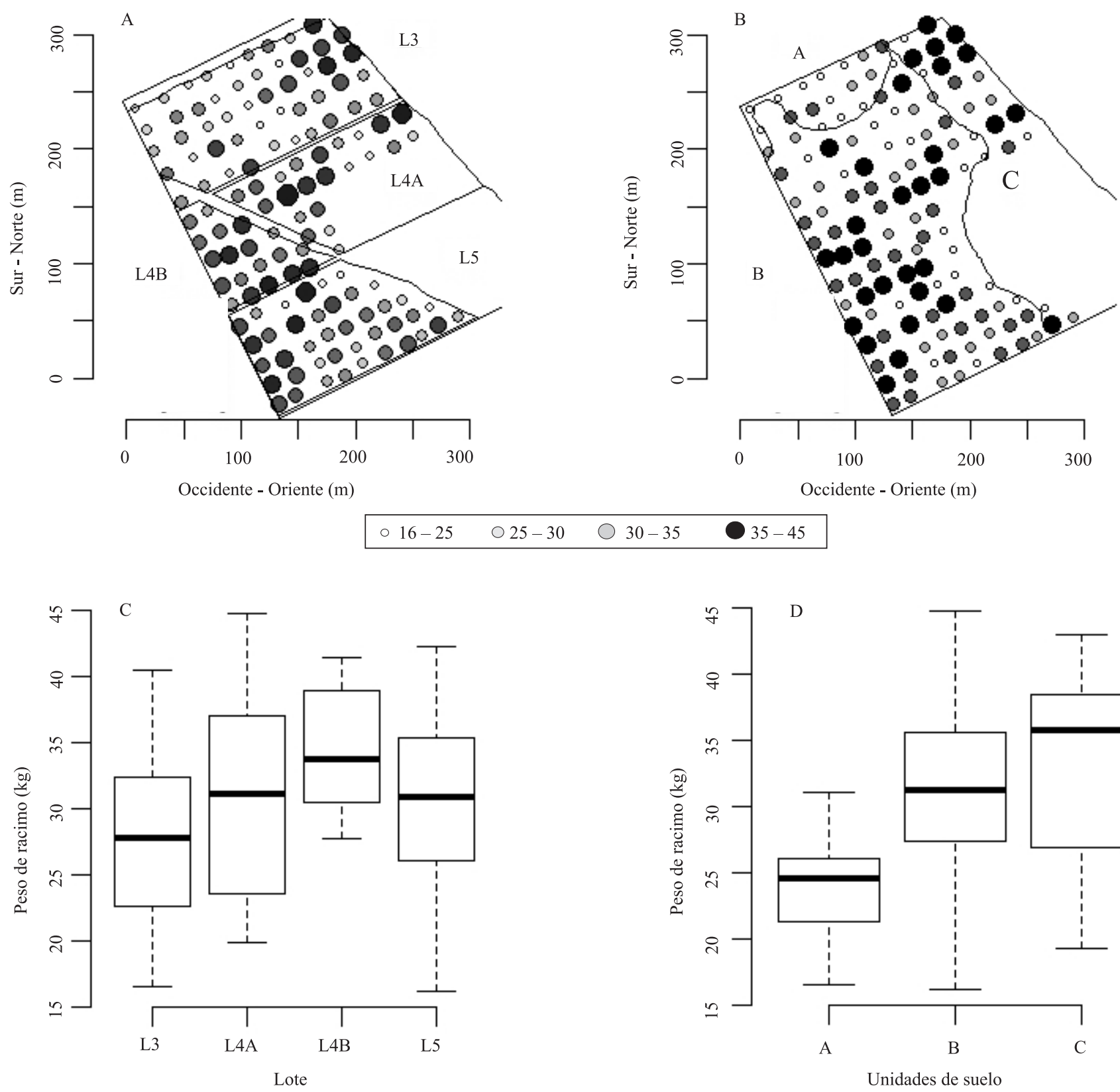

$25-30 \bigcirc 30-35 \bigcirc 35-45$ 
la unidad de suelo A es diferente de las unidades B y C $(p=0,029$ y 0,018 respectivamente), pero no lo es entre las unidades B y C ( $>0,05)$. Estos resultados indican que, dependiendo de la manera como se agrupen los pesos de los racimos dentro del área de estudio, el nivel de variabilidad entre ciertos grupos es diferente.

En el área de estudio, el peso promedio de los racimos fue de $30,41 \mathrm{~kg}$, y varió entre 16,1 y $44,7 \mathrm{~kg}$, con promedios de $28,09,31,14,34,29$ y $30,85 \mathrm{~kg}$ para los lotes L3, L4A, L4B y L5, respectivamente, y de 24,26, 30,96, y 32,82 kg para las unidades A, B y C en su orden. Presumiéndose varianzas no homogéneas, hubo diferencia significativa estadísticamente sólo entre los lotes L3 y L4B ( $p<0,001)$; en los demás casos, el valor de $\mathrm{p}$ fue mayor a 0,05 . Respecto a las unidades de suelos, el peso promedio de los racimos de la unidad A fue significativamente diferente del de las unidades B y $\mathrm{C}(\mathrm{p}=0,0004$ y $\mathrm{p}=0,0003$, respectivamente) y no hubo diferencia entre las unidades B y C $(p=0,470)$ (Figuras 1 $\mathrm{C}$ y D). Lo anterior evidencia la existencia de sectores dentro de la finca con diferentes productividades, similarmente a lo reportado por Serrano et al. (2006), y que, además, pueden ser identificados y delimitados a través de características fisiográficas en campo, asociadas al manejo, como son los límites de los lotes.

El análisis de tendencia espacial para el peso del racimo, en función de los ejes coordenados, produce la siguiente superficie de tendencia lineal: $P R=48,04-0,01 d_{1}-0,01 d_{2}+0,001 d_{1} d_{2}\left(R^{2}\right.$ ajustado $=0,169$ y valor $p=0,00766$ ). La superficie anterior explica el 16,9\% del comportamiento del peso de los racimos dentro del campo (Cuadro 1), lo que lo hace un modelo inadecuado para este propósito, dato que corrobora lo reportado por Diggle y Ribeiro Junior (2007), respecto a la comparación del variograma de los residuales, una vez extraída la tendencia con dos variogramas empíricos envolventes. Los variogramas son generados por la selección de varianzas máximas y mínimas, calculadas a partir de 99 simulaciones aleatorias con los parámetros del modelo ajustado en cada ubicación. La evaluación indicó que los residuales continúan a conservar una estructuración espacial significativa y similar a la de los datos originales.

La tendencia espacial en función de las covariables, para todo el campo experimental, de las variables edáficas evaluadas, sólo se correlacionó significativamente con el peso del racimo, los contenidos de arena, de Ca y de $\mathrm{Mg}$, el pH, la H15, el DPMS y el DPMH. El modelo que los relacionó tuvo valor $\mathrm{p}<0,0000319$ y $\mathrm{R}^{2}$ ajustado del $28,3 \%$. Aunque con este modelo se mejora la estacionaridad del peso de los racimos, en el campo de estudio, su capacidad predictiva sigue siendo baja, haciéndolo un modelo poco prometedor, en términos de explicar su comportamiento.

En la clase de suelo A, el grupo de variables que se relacionó significativamente con el peso del racimo permitió explicar el 50,1\% del comportamiento de este, mientras que en las clases $\mathrm{B}$ y $\mathrm{C}$, los niveles de explicación de los diferentes grupos de variables fueron del 29,6 y $28,8 \%$, respectivamente. No obstante el comportamiento mencionado, hay dos aspectos relevantes: el primero tiene que ver con la diferencia en los grupos de covariables que se relacionaron con el peso de los racimos, en cada clase de suelo. El segundo aspecto es la persistencia de la tendencia en el peso de los racimos, dentro de la unidad de suelos B, donde se observaron sectores definidos con pesos de racimos altos y sectores con pesos bajos, lo que puede indicar que las unidades de suelos, delimitadas en este campo, no discriminan adecuadamente la tendencia espacial presentada en la producción de banano, probablemente debido a que la escala (1:5.000), a la cual son realizados estos trabajos, sobrepasa las distancias a las cuales se presenta la tendencia espacial en la producción. De manera similar, Brejda et al. (2000) buscaron estimar la probabilidad de distribución de las varibales del suelo y encontraron que aun agrupando los muestreos por series de suelos, las estimaciones tenían una baja probabilidad, inducida por una alta variabilidad en las series.

Los coeficientes de determinación para el análisis del peso del racimo, con las variables del suelo evaluadas como covariables y de acuerdo a los lotes, fueron de $96,9,95,6,84,1$ y $68,9 \%$ para los lotes L3, L4A, L4B y L5, respectivamente, lo que evidencia un mejor ajuste e indica que los lotes son una mejor alternativa para extraer la tendencia. El ajuste entre el peso real de los racimos de banano con los valores estimados, usándose las variables y parámetros del Cuadro 1, mejoró cuando se usaron los lotes como factor de control de la tendencia espacial (Figura 2). Este mejor ajuste, probablemente, se debe a que el manejo agronómico de las fincas bananeras está definido a través de los lotes, y que - como lo sugieren Paz-González et al. (2000) - parte de la variabilidad de 


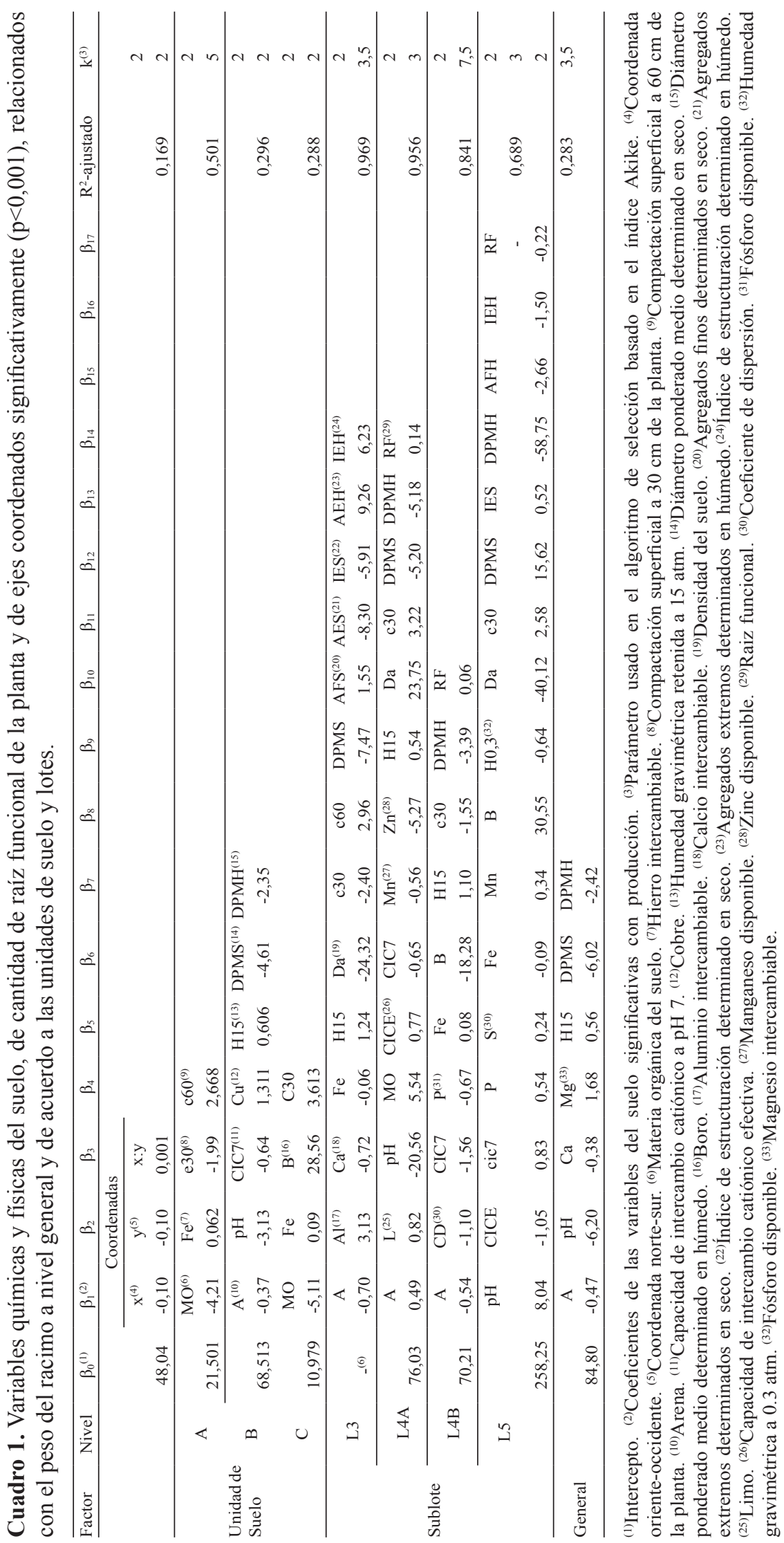

Pesq. agropec. bras., Brasília, v.45, n.8, p.836-845, ago. 2010 
los suelos está asociada a condiciones inducidas por el uso y manejo de ellos.

Aunque los lotes definieron adecuadamente los sitios de tendencias existentes, estos no son una característica directamente relacionada con la producción, lo que parecería indicar que estas áreas están determinadas por el grupo de variables relacionado con el peso del racimo identificado dentro de cada lote y que, además, es diferente entre lotes. Debido al aporte individual e interactivo a la variabilidad total del grupo de características significativas y diferentes entre lotes, se minimiza la respuesta con dos o tres variables, analizadas en áreas mayores. Quizás esta sea la razón de que, al realizar una estrategia de manejo agronómica homogénea para todo un predio, la respuesta en producción sea baja.

La función media del peso de los racimos, para toda el área estudiada en función de los lotes como factor de extracción de la tendencia y de las covariables físicas y químicas del suelo y de raíz funcional, relevantes en cada uno de ellos, explica el peso de los racimos de banano en un 97,5\%, con un valor $p<0,0001$ (Figura $3 \mathrm{~A}$ ) y presenta un ajuste muy superior al obtenido inicialmente de $17 \%$. Los residuales obtenidos por la diferencia entre los valores reales de peso y los estimados a través del modelo completo muestran una distribución aleatoria dentro del lote y sin tendencias (Figura 3 B).

El campo aleatorio de la producción de banano presenta componentes de variabilidad a diferentes escalas de variación dependientes de la dirección, evidenciado en la proporción y rango presentados por los variogramas para los diferentes ángulos del peso de los racimos (Cuadro 2). La dependencia espacial es débil para los ángulos de $0^{\circ}$ y $90^{\circ}$ mayor que 0,8 y es moderada para las demás direcciones (Cambardella et al., 1994). Lo anterior parece estar relacionado con el efecto de variables de diferentes rangos en el campo de cultivo de banano,

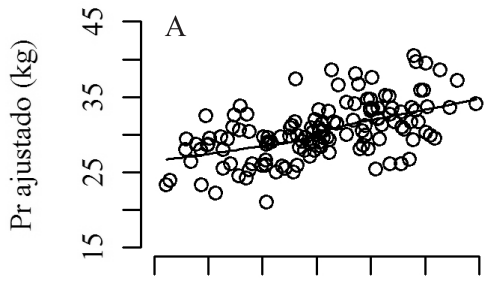

$\begin{array}{llll}15 & 25 & 35 & 45\end{array}$
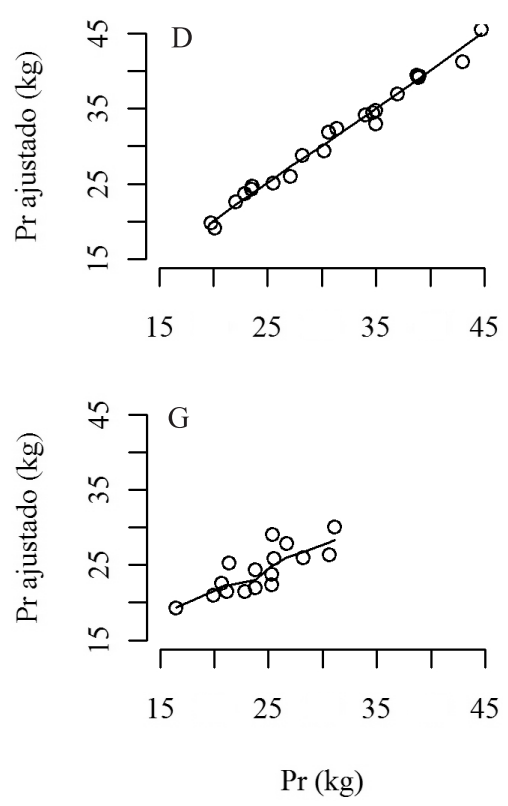
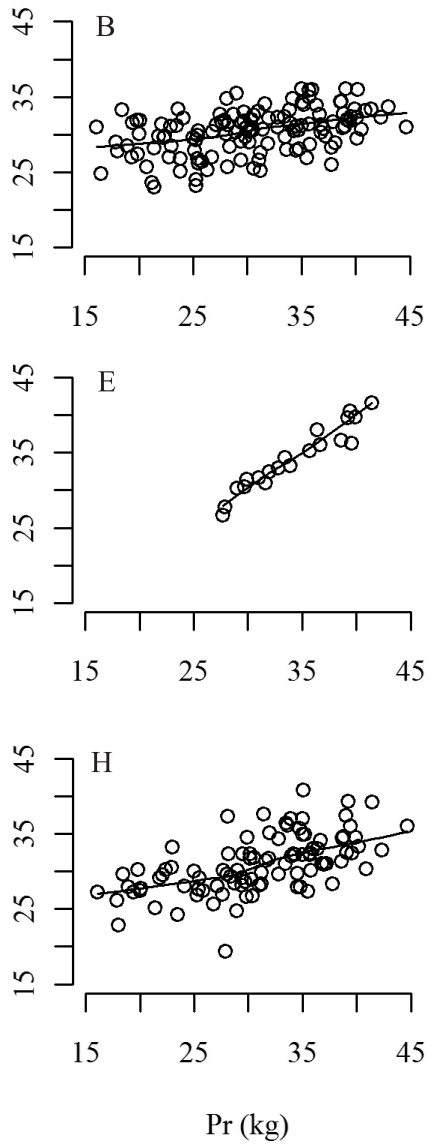

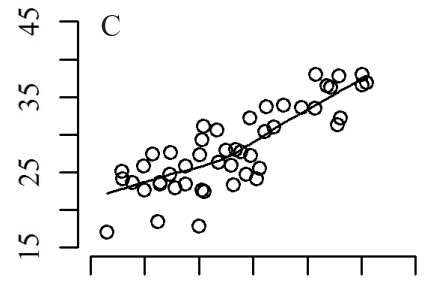

$\begin{array}{llll}15 & 25 & 35 & 45\end{array}$
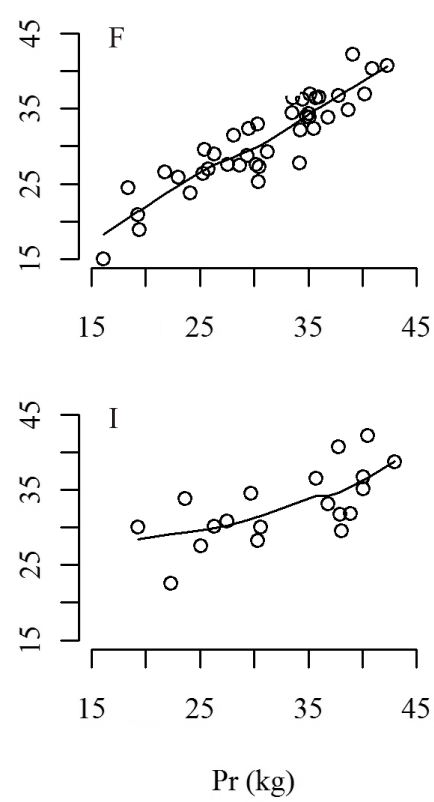

Figura 2. Ajuste entre el peso de los racimos de banano (Pr) y valores de peso predichos a partir del grupo de variables explicativas significativas en: A, todo el área investigada; B, con los ejes coordenados; C, lote 3; D, lote 4A; E, lote 4b; F, lote 5; G, unidad de suelo A; H, unidad de suelo B; I, unidad de suelo C. 
cuyas escalas son dependientes de la dirección, características típicas de un proceso no estacionario y anisotrópico. Este hallazgo concuerda con los cambios de pendiente, encontrados en los semivariogramas reportados por Bourennane et al. (2004), de algunas

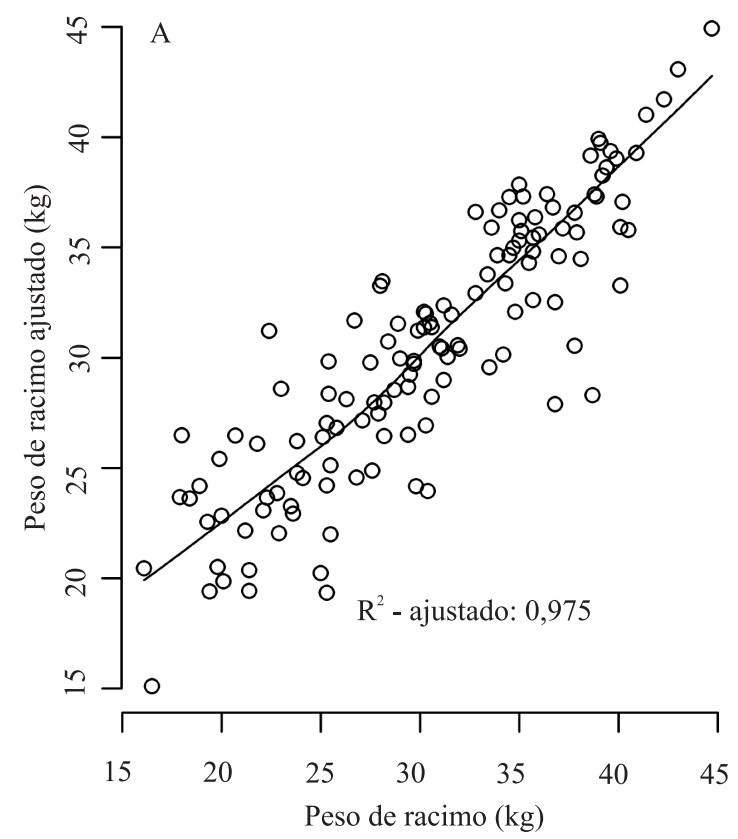

variables del suelo asociadas con la producción de trigo y que ellos relacionaron con posibles tendencias espaciales en estas variables. El ajuste (relación de anisotropia de 3 ) y la rotación $\left(60^{\circ}\right)$ de los ejes hacen que el promedio en el peso de los racimos no dependan

Figura 3. Extracción de la tendencia espacial para el peso de los racimos. A, ajuste general entre el peso real y estimado de los racimos, para el modelo general en función de variables explicativas del suelo significativas según el lote; B, gráfico de burbujas para los residuales del modelo ajustado. El tamaño y el color del círculo están asociados con el peso del racimo.

Cuadro 2. Parámetros de los modelos ajustados a los semivariogramas empíricos isotrópicos y anisotrópicos, generados con los datos georreferenciados del peso de los racimos y con los residuales de la diferencia entre el peso real y estimado de los racimos, por el modelo general de predicción en función de variables explicativas del suelo, significativas según el lote.

\begin{tabular}{|c|c|c|c|c|c|c|c|}
\hline Ángulo & Modelo & Pepita & Meseta & $\varphi^{(1)}$ & $\mathrm{Kappa}^{(2)}$ & Proporción ${ }^{(3)}$ & Alcance \\
\hline & & \multicolumn{6}{|c|}{ Peso del racimo } \\
\hline Isotrópico & Matern & 20,00 & 32,13 & 35,90 & 0,50 & 0,62 & 107,56 \\
\hline $0^{\circ}$ & Matern & 25,00 & 31,97 & 16,77 & 3,00 & 0,88 & 107,63 \\
\hline $30^{\circ}$ & Matern & 25,00 & 50,81 & 68,97 & 2,00 & 0,49 & 275,79 \\
\hline $60^{\circ}$ & Matern & 20,00 & 39,41 & 36,65 & 1,00 & 0,51 & 146,56 \\
\hline $90^{\circ}$ & Matern & 25,00 & 27,71 & 18,54 & 2,00 & 0,90 & 99,51 \\
\hline \multirow{2}{*}{ Rotado $\left(\operatorname{Ang}^{(4)}=60^{\circ}, \mathrm{RA}^{(5)}=3\right)$} & Exponencial & 24,84 & 37,13 & 47,99 & 0,50 & 0,67 & 430,67 \\
\hline & & \multicolumn{6}{|c|}{ Residuales } \\
\hline Isotrópico & Pepita puro & $-(6)$ & 6,60 & - & - & - & - \\
\hline $0^{\circ}$ & Exponencial & 3,50 & 3,85 & 25,13 & 0,50 & 0,91 & 75,28 \\
\hline $30^{\circ}$ & Matern & 2,50 & 2,60 & 13,81 & 1,00 & 0,96 & 55,21 \\
\hline $60^{\circ}$ & Pepita puro & - & 6,23 & - & - & - & - \\
\hline $90^{\circ}$ & Matern & 2,31 & 5,83 & 22,31 & 1,00 & 0,40 & 89,22 \\
\hline Rotado $\left(\right.$ Ang $\left.=90^{\circ}, \mathrm{RA}=3\right)$ & Exponencial & 2,50 & 4,58 & 7,34 & 2,00 & 0,55 & 12,70 \\
\hline
\end{tabular}

${ }^{(1)}$ Tasa a la cual la correlación decae a cero, cuando se aumenta la distancia entre dos puntos. ${ }^{(2)}$ Parámetro de forma de la función de correlación. ${ }^{(3)}$ Relación entre el efecto pepita y la semivarianza total; valores $<0,25$ indican una fuerte dependencia espacial, entre 0,25 y 0,75 indican una moderada dependencia espacial y valores $>0,75$ indican una débil dependencia espacial (Cambardella et al., 1994). ${ }^{(4)}$ Ángulo de rotación de los ejes. ${ }^{(5)}$ Relación de anisotropía. ${ }^{(6)}$ No se aplica para el modelo pepita puro. 
de la dirección (Cuadro 2), lo que genera un variograma con los siguientes parámetros: pepita, 24,84; meseta, 37,13 y un rango de 430 . Las covariables que fueron significativas dentro de cada lote extraen y explican más del $80 \%$ de la variabilidad espacial total, con respecto al no uso de covariables, evidenciado en la menor magnitud de los parámetros pepita, meseta y proporción en los variogramas ajustados a los residuales, respecto a las variables originales (Cuadro 2). Aún queda un efecto anisotrópico en los residuales, para la dirección de $90^{\circ}$ con una dependencia espacial moderada.

Finalmente, se considera que la descomposición de la variabilidad efectuada y representada en el semivariograma experimental encontrado es técnica y científicamente relevante, puesto que la función media de la producción del área estudiada está explicada en función de variables que tienen significado agronómico, y se la sugiere como soporte en el planteamiento de una estrategia de manejo por sitio específico en el cultivo de banano.

\section{Conclusiones}

1. Para las condiciones estudiadas, los lotes son la característica fisiográfica que permite una mejor descomposición de la variabilidad espacial de la producción de banano.

2. Las características edáficas relacionadas con producción de banano difieren entre lotes y son las que mayor potencial tienen para el establecimiento de un programa de manejo por sitio específico.

3. Las clases de suelono se relacionan con la variabilidad espacial, en la producción de banano, ni controlan las propiedades del suelo que se relacionan con esta.

4. El variograma experimental encontrado integra propiedades químicas y físicas que permiten explicar más del $80 \%$ de la variabilidad espacial de la producción de banano.

\section{Agradecimientos}

Al Dirección de Investigación Medellín de la Universidad Nacional de Colombia, al Ministerio de Agricultura de Colombia, a la Asociación de Bananeros de Colombia, por la cofinanciación del proyecto; y al Laboratorio de Suelos de la Escuela de Geociencias de la Universidad Nacional de Colombia, Sede Medellín, por su colaboración.

\section{Referencias}

AMADO, T.J.C.; PONTELLI, C.B.; SANTI, A.L.; VIANA, J.H.M.; SULZBACH, L.A. de S. Variabilidade espacial e temporal da produtividade de culturas sob sistema plantio direto. Pesquisa Agropecuária Brasileira, v.42, p.1101-1110, 2007.

ARAYA-VARGAS, M. Metodología utilizada en el laboratorio de nematología de Corbana S.A. para la extracción de nematodos de las raíces de banano (Musa AAA) y plátano (Musa AAB). CORBANA, v.28, p.97-110, 2002.

BLACKMORE, S. The interpretation of trends from multiple yield maps. Computers and Electronics in Agriculture, v.26, p.37-51, 2000.

BOURENNANE, H.; NICOULLAUD, B.; COUTURIER, A.; KING, D. Exploring the spatial relationships between some soil properties and wheat yields in two soil types. Precision Agriculture, v.5, p.521-536, 2004.

BREJDA, J.J.; MOORMAN, T.B.; SMITH, J.L.; KARLEN, D.L.; ALLAN, D.L.; DAO, T.H. Distribution and variability of surface soil properties at a regional scale. Soil Science Society of America Journal, v.64, p.974-982, 2000.

CALVO, C.; ARAYA-VARGAS, M. Cantidad de raíces de banano en los diez cantones productores de Costa Rica. CORBANA, v.27, p.47-64, 2001.

CAMBARDELLA, C.A.; MOORMAN, T.B.; NOVAK, J.M.; PARKING, T.B.; KARLEN, D.L.; TURCO, R.F.; KONOPKA, A.E. Field-scale variability of soil properties in central Iowa soils. Soil Science Society of America Journal, v.58, p.1501-1511, 1994.

DICKER, K.; HERMANN, D.F.; BRODAHL, M.K. Frequency analysis of yield for delineating yield response zones. Precision Agriculture, v.5 p.435-444, 2004.

DIGGLE, P.J.; RIBEIRO JUNIOR, P.J. Model-based geostatistics. New York: Springer, 2007. 228p.

GAUGGEL, C.A.; SIERRA, F.; ARÉVALO, G. The problem of the banana root deterioration and its impact on production: Latin America's experience. In: INTERNATIONAL SYMPOSIUM, 2003, San José. Banana root system: towards a better understanding for its productive management. San Jose: INIBAP, 2003. p.13-22.

JARAMILLO, D.F.J. Efecto de la variabilidad sistemática de la producción de frijol en experimentos de fertilización. Segunda siembra. Revista Facultad Nacional de Agronomia, v.59, p.31-47, 2006.

KRAVCHENKO, A.N.; BULLOCK, D.G. Correlation of corn and soybean grain yield with topography and soil properties. Agronomy Journal, v.92, p.75-83, 2000.

LAMBERT,D.M.;LOWEMBERG-DEBOER,J.;BONGIOVANNI, R. A comparison of four spatial regression models for yield monitor data: a case study from Argentina. Precision Agriculture, v.5, p.579-600, 2004.

LARK, R.M. Optimized spatial sampling of soil for estimation of the variogram by maximum likelihood. Geoderma, v.105, p.49-80, 2002.

LONG, D.S. Spatial autoregression modeling of site-specific wheat yield. Geoderma, v.85, p.181-197, 1998. 
MIAO, Y.; MULLA, D.J.; ROBERT, P.C. Spatial variability of soil properties, corn quality and yield in two Illinois, USA fields: implications for precision corn management. Precision Agriculture, v.7, p.5-20, 2006.

MOTTA, M.B. Métodos analíticos del laboratorio de suelos. 5.ed. Bogotá: IGAC, 1990. 502p.

PAZ-GONZÁLEZ, A.; VIEIRA, S.R.; CASTRO, M.T.T. The effect of cultivation on the spatial variability of the selected properties of an umbric horizon. Geoderma, v.97, p.273-292, 2000.

PING, J.L.; DOBERMANN, A. Processing of yield map data. Precision Agriculture, v.6 p.193-212, 2005.

R DEVELOPMENT CORE TEAM. R: a language and environment for statistical computing. Vienna: R Foundation for Statistical Computing, 2009.

RIBEIRO JUNIOR, P.J.; DIGGLE, P.J. geoR: a package for geostatistical analysis. R News, v.1, p.15-18, 2001.

SADLER, E. J.; SUDDUTH, K. A.; JONES, J. W. Separating spatial and temporal sources of variation for model testing in precision agriculture. Precision Agriculture, v.8, p.297-310, 2008.
SERRANO, E.; SANDOVAL, J.; POCASANGRE, L.; ROSALES, F.; DELGADO, E. Importancia de los indicadores físico-químicos en la calidad del suelo para la producción sustentable del banano en Costa Rica. In: REUNIÃO INTERNACIONAL ACORBAT, 17., 2006, Joinville. Bananicultura: um negócio sustentável: anais. Joinville: ACORBAT: ACAFRUTA, 2006. p.207-221.

STOORVOGEL, J.; VARGAS, R. La agricultura de precisión en banano. In:ROSALES-IZAGUIRRE, F.E.;TRIPON, S.C.; CERNA, J. (Ed.). Producción de banano orgánico y/o ambientalmente amigable. Turrialba: CATIE, 1998. p.40-55.

UNITED STATES DEPARTMENT OF AGRICULTURE. Keys to soil taxonomy. $10^{\text {th }}$ ed. Washington, D. C.: USDA, 2006. 332p.

VOORTMAN, R.L.; BROUWER, J. An empirical analysis of the simultaneous effects of nitrogen, phosphorus, and potassium in millet production on spatially variable fields in SW Niger. Nutrient Cycling in Agroecosystems, v.66, p.143-164, 2003.

ZHANG, N.Q.; WANG, M.H.; WANG, N. Precision agriculture: a worldwide overview. Computers and Electronics in Agriculture, v.36, p.113-13, 2002.

Recibido el 2 de junio de 2009 e aprobado el 15 de julio de 2010 